\title{
Erratum to: On Gauss-type quadrature formulas with prescribed nodes anywhere on the real line
}

\author{
Adhemar Bultheel · Ruymán Cruz-Barroso • \\ Marc Van Barel
}

Published online: 1 May 2013

(C) Springer-Verlag Italia 2013

\section{Erratum to: Calcolo (2010) 47:21-48 \\ DOI 10.1007/s10092-009-0013-x}

In the paper [2] we investigated among other things the existence of Gauss-type quadrature formulas with two prescribed nodes and a maximal domain of exactness. At some instances we claimed that the weights in the quadrature formulas are positive, while this only holds under more restrictive conditions than given. These are the corrections that have to be implemented.

1. In the discussion before Theorem 2.15, the case GL3c should be completed with the following additional information.

$T(b)=f_{n}(b)$ iff $a$ is a node of the quadrature and $T(a)=f_{n}(a)$ iff $b$ is a node. If all the nodes are in $(a, b)$ the corresponding weights will be positive. If $a$ or $b$ is one of the nodes, then the corresponding weight cannot be guaranteed to be positive.

The online version of the original article can be found under doi:10.1007/s10092-009-0013-x.

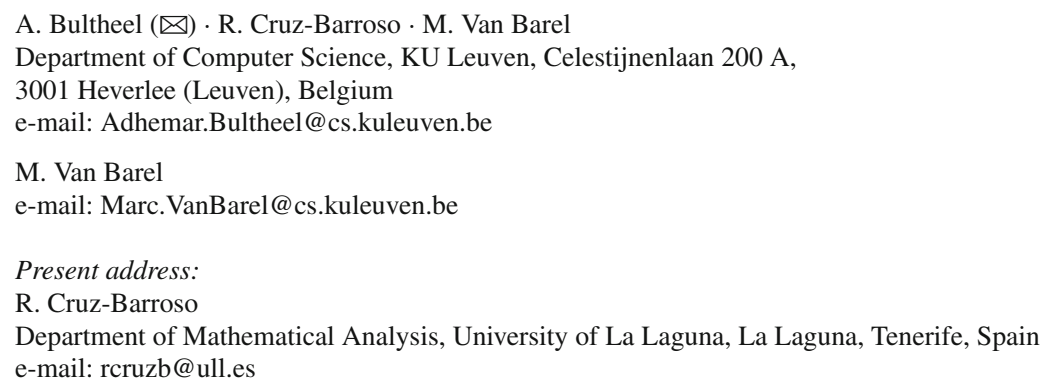


2. The last sentence of Theorem 2.15 should be changed as follows.

In all the cases where the Gauss-Lobatto quadrature rule exist, it must be of interpolatory type and the positivity of the weights for all the nodes inside $(a, b)$ is guaranteed. The positivity of the weight corresponding to a node in one of the endpoints of the interval cannot be guaranteed.

3. In Corollary 2.17, the sentence "The weights of the quadrature are positive" should be replaced by the following:

The weights corresponding to the nodes different from $a$ if $\tilde{\mu}(x)=(x-a) \mu(x)$ (respectively different from $b$ if $\tilde{\mu}(x)=(b-x) \mu(x))$ are positive, but the weight for $a$ (respectively $b$ ) cannot be guaranteed to be positive.

More details about conditions that guarantee that all the weights are positive are provided in [1].

Acknowledgments We thank Bernd Beckermann for pointing this out to us and for providing us with the manuscript of [1].

\section{References}

1. Beckermann, B., Bustamante, J., Martínez-Cruz, R., Quesada, J.M.: Gaussian, Lobatto and Radau positive quadrature rules with a prescribed abscissa (2013)

2. Bultheel, A., Cruz-Barroso, R., Van Barel, M.: On Gauss-type quadrature formulas with prescribed nodes anywhere on the real line. Calcolo 47(1), 21-48 (2010) 